\title{
Thermal and aerodynamic tests of a digital combined current and voltage transformer
}

\author{
Sergey Litvinov ${ }^{1}$, Vladimir Lebedev ${ }^{1}$, Nikolay Smirnov, ${ }^{2, *}$, Vladimir Tyutikov ${ }^{2}$ and Sergey \\ Shuvalov ${ }^{2}$ \\ ${ }^{1}$ Ivanovo State Power Engineering University, Electric Power Engineering Department, 153003 \\ Ivanovo, Russia \\ ${ }^{2}$ Ivanovo State Power Engineering University, Heat Power Engineering Department, 153003 Ivanovo, \\ Russia
}

\begin{abstract}
This study examines the results of thermal and aerodynamic tests of a digital combined current and voltage transformer conducted in an environmental chamber. This measuring instruments consist of current and voltage transformers, featuring a resistive divider, and are used for commercial and technical electric power accounting. Different ambient temperatures, airflow rates and levels of insolation were set for the environmental chamber, with simulation of transformer functioning in emergency modes. It was established that heat release at transformer outer surface with natural convection depends to a larger extent on the difference between the temperature at the transformer surface and ambient temperature, while with forced convection this heat release depends more on air mass speed, with greater heat release on the surface of an upward facing rib than on the surface of a downward facing rib. The results of our study have been used in developing algorithms for diagnostics of the thermal state of digital combined transformers.
\end{abstract}

\section{Introduction}

An important task in the functioning and thermal overload protection [1] of digital combined current and voltage transformers in commercial and technical electric power accounting in summer conditions with high ambient temperatures and levels of insolation and the possibility of grid voltage exceeding rated values is to examine the exchange of heat between a transformer and its environment. The main methods of thermal overload protection are widely known [1-3]. An important indicator in research conducted with the aid of software enabling simulation of heat exchange between a transformer and its environment following changes in initial conditions is the accuracy of the resulting data. Laboratory research conducted with physical models of digital transformers is one of the best methods of verifying such models.

We conducted a series of experiments in the laboratories of two of our departments (High Voltage Equipment and Industrial Heat Power Engineering) to study the influence of voltage, high ambient temperatures and insolation on the operating temperature of a new

\footnotetext{
*Corresponding author: nsmirnov@,bk.ru
} 
design of digital combined transformer, and also to determine the destructive temperature for resistors in unfavourable conditions. We studied the dynamics of the processes involved.

The digital instrument transformer consists of a resistive voltage divider and current transformer (see Figure 1). Electrical processes occurring in resistive voltage dividers comprising groups of resistors are related to heat emission [3]. Resistor overheating may result in measuring equipment failure.

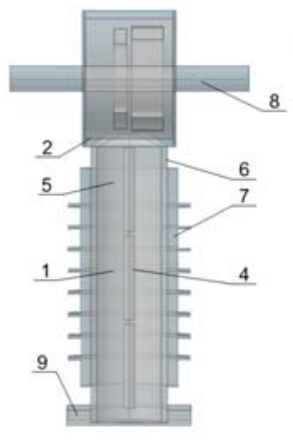

a)

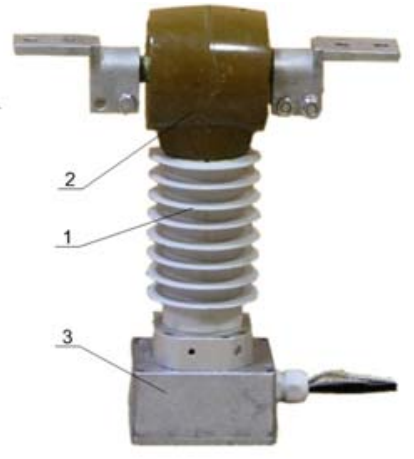

b)

Fig. 1. Diagram (a) and external view (b) of a 6-10kW digital combined transformer: 1 - primary voltage converter; 2 - primary current power converter; 3 - low voltage electronic unit; 4 - resistive divider; 5 - silicon filling; 6 - glass textolite body; 7 - cover; 8 - copper rod; 9 - metal flange.

\section{Testing in a high-voltage environmental chamber}

A part of our research was conducted in an environmental chamber designed for highvoltage testing. The diagram and general view of this chamber with installed equipment are shown in Figure 2. The environmental chamber comprises a closed, isolated structure consisting of two compartments: a warm compartment and one for a step-up voltage transformer. The warm compartment is shielded by means of PVC sheeting, and features a heating element used to maintain constant ambient temperature, creating the necessary temperature conditions corresponding to the worst transformer operating conditions, taking into account the environmental version and placement category.

An electrode was introduced into the chamber to supply high voltage to the test item from the laboratory UIV- $150 \mathrm{~kW}$ unit, consisting of a control panel and step-up transformer. Ambient temperature and humidity, also temperature at the resistors, were determined by means of sensors. A TESTO-875-1i thermal imager was also used to determine transformer surface temperature fields. Insolation on the collecting surface of the warm compartment in the chamber was simulated using a $250 \mathrm{~W}$ incandescent filament lamp located on a device enabling us to change and fix lamp angle of elevation. Preliminary experiments were conducted to calibrate the values of radiation energy emitted from the incandescent filament lamp onto the collecting surface.

A resistive voltage divider functions at various thermal conditions, and the temperature (as well as the number of temperature cycles) significantly influences both the metrological properties of the resistor, and its performance. In the preliminary experiment, we determined the temperature of resistor destruction or loss of its main properties in air. As a scale analogue, we used a resistive divider of $6(10) \mathrm{kW}$ voltage class, taking emergency mode as the initial mode, with supplied voltage exceeding the nominal value 2.1 times and $U=4.4 \mathrm{~kW}$. Resistance in the sensor $R_{s}$ was $10 \mathrm{kOhm}$ at $25^{\circ} \mathrm{C}$, within $1 \%$. A temperature 
sensor (B57861S) was connected to the lower contact cap of the resistor via a thermal interface (see Figure 3).
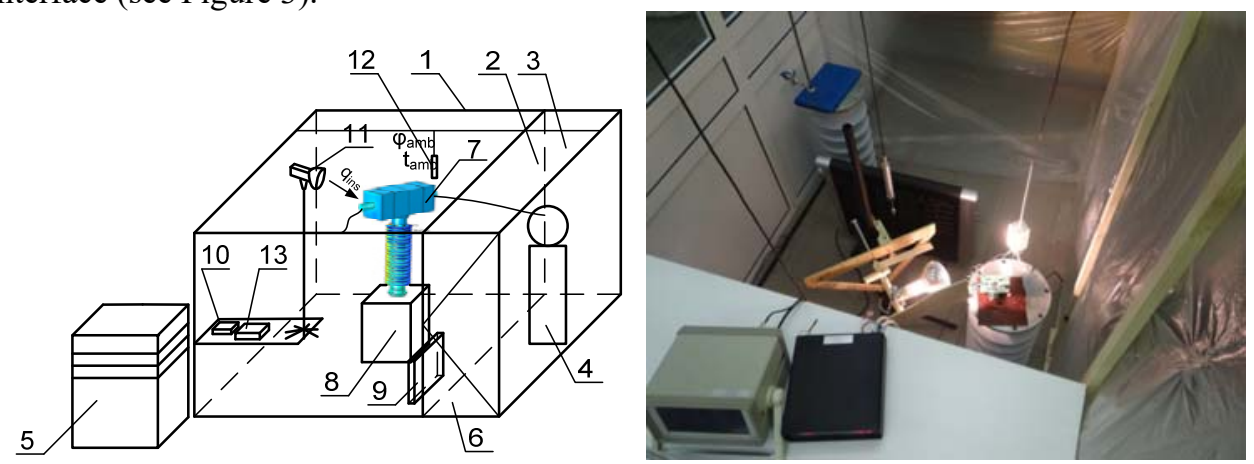

Fig. 2. Diagram of an environmental chamber in a high-voltage test laboratory: 1 - warm compartment; 2 - partition; 3 - step-up transformer compartment; 4 - step-up transformer; 5 - control panel; 6 - compartment door; 7 - test item; 8 - earthed prop for the test item; 9 - heating element; 10 - temperature measuring and controlling device; 11 - incandescent-filament lamp for simulating insolation; 12 - primary humidity and temperature converter; 13 - thermal hygrometer.
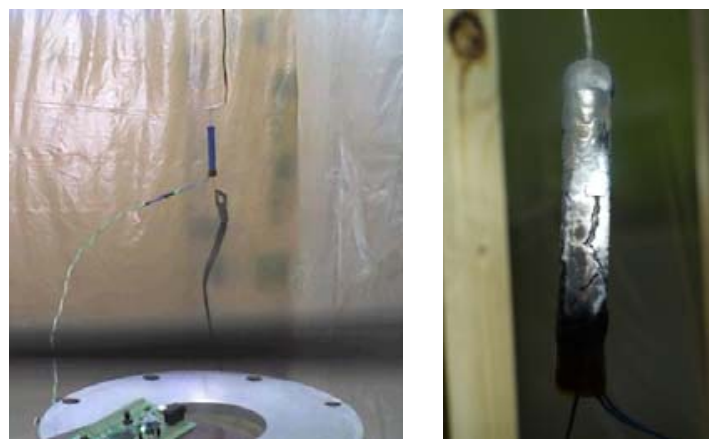

Fig. 3. General view of the test item before energization and after testing (charred).

Insulation and cooling were provided by air, with no insolation. A constant temperature was maintained inside the environmental chamber, with ambient temperature $t_{a m b}=36^{\circ} \mathrm{C}$.

During the test, resistor surface temperature exceeded the set value within 18 minutes (see Figure 4), after which the supplied voltage increased in steps of $1 \mathrm{~kW}$ with time delay up to the settled mode, destruction of the resistor or loss of its main properties. With voltage of $7.1 \mathrm{~kW}$ and a temperature of around $200^{\circ} \mathrm{C}$ the resistor outer layer began to char, while with a temperature of around $240{ }^{\circ} \mathrm{C}$ smoke emission began, its intensity growing with increasing temperature. The resistor began to lose its main properties: output voltage of the device began to fall owing to the formation of a superficial conducting layer. A temperature of around $340{ }^{\circ} \mathrm{C}$ resulted in combustion of the resistor, after which the experiment was stopped.

After completing the experiment, we measured resistor resistance, which amounted to $40 \mathrm{M} \mathrm{Ohm}$. The critical resistor operation temperature is taken to be $200^{\circ} \mathrm{C}$, but since the resistor in a digital transformer is placed within a silicon filling, this critical temperature should be lowered to $150{ }^{\circ} \mathrm{C}$ (temperature at which silicon destruction commences).

After determining the permissible temperatures for operation of a single resistor we also tested a resistor placed in silicon insulation (see Figure 5), with different voltages and simulated standard ambient temperature within $38-40{ }^{\circ} \mathrm{C}$ (with the help of a heater) and insolation within 1000-1100 W/m² (using an incandescent filament lamp). 
As shown by experimental data of examining the thermal state of a resistor, supplied voltage and insolation significantly influence the object's working temperature.

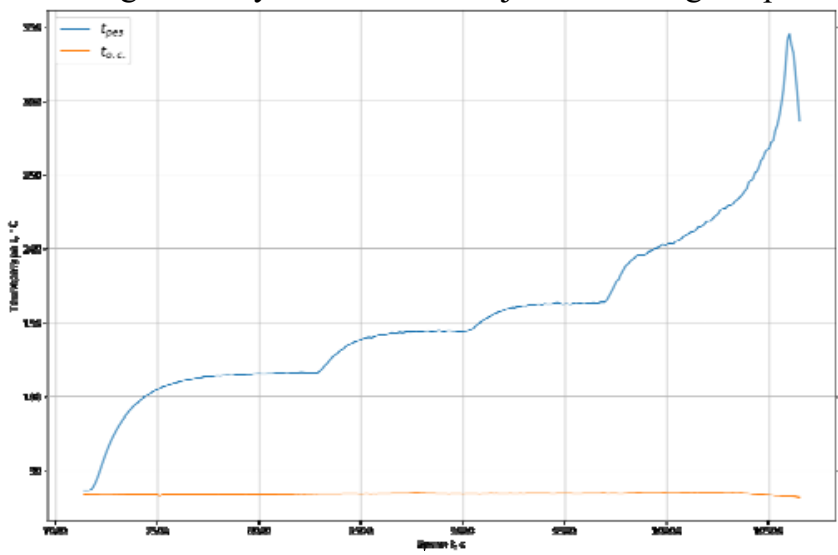

Fig. 4. Charts showing dependence of ambient temperature and temperature in resistor lower contact cap on time.

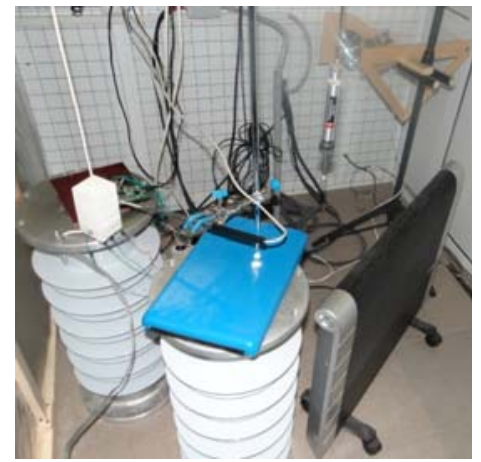

a)

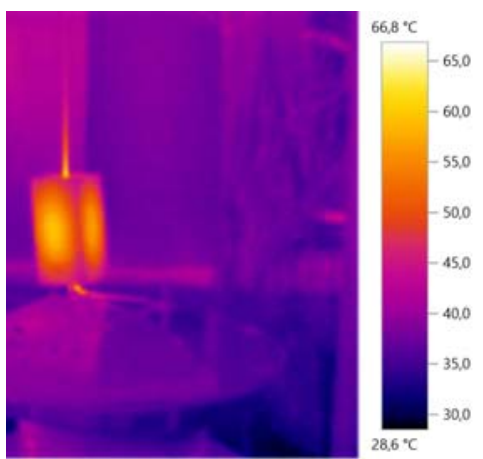

b)

Fig. 5. General view of testing system (a) and thermograph. (b) for a resistor in a silicon casing.

The first series of experiments was conducted in the absence of insolation. With nominal voltage (see Figure 6, a) in one resistor $U_{\text {nom }}=1.92 \mathrm{~kW}$, the resistor heated up to a temperature of $t_{r}=59{ }^{\circ} \mathrm{C}$ in 1 hour 55 minutes, while surface temperature rose to $t=41{ }^{\circ} \mathrm{C}$. With voltage of $1,2 \mathrm{x} U_{\text {nom }}=2.31 \mathrm{~kW}$, the resistor heated up to a temperature of $t_{r}=67,5{ }^{\circ} \mathrm{C}$ in 2 hours 25 minutes, while surface temperature rose to $t=43,5{ }^{\circ} \mathrm{C}$. With emergency voltage of $2,1 \times U_{n o m}=4.04 \mathrm{~kW}$, the resistor heated up to a temperature of $t_{r}=114{ }^{\circ} \mathrm{C}$ in 2 hours 5 minutes, while surface temperature rose to $t=48.5^{\circ} \mathrm{C}$.

In the second series of experiments with simulated insolation (see Figure $6, \mathrm{~b}$ ) and nominal voltage $U_{\text {nom }}=1.92 \mathrm{~kW}$, the resistor heated up to a temperature of $t_{r}=79.1^{\circ} \mathrm{C}$ in 2 hours, while the temperature of the lamp facing surface rose to $t=110^{\circ} \mathrm{C}$. At this voltage, insolation further raised the temperature in the resistor by $\Delta t=20^{\circ} \mathrm{C}$, and the temperature of the silicon surface facing the lamp by $\Delta t=69^{\circ} \mathrm{C}$.

With a voltage of $1,2 \mathrm{x} U_{\text {nom }}=2.31 \mathrm{~kW}$, the resistor heated up to a temperature of $t_{r}$ $=83.1{ }^{\circ} \mathrm{C}$ in 2 hours 5 minutes, while insolation further raised resistor temperature by $\Delta t=15.6^{\circ} \mathrm{C}$.

With emergency voltage of $2,1 \times U_{\text {nom }}=4.03 \mathrm{~kW}$, the resistor heated up to a temperature of $t_{r}=131{ }^{\circ} \mathrm{C}$, while the temperature of the lamp facing surface rose to $t=112^{\circ} \mathrm{C}$. We can see that in this case, resistor temperature is higher than the temperature of the insulation 
surface. Insolation further raised the temperature in the resistor by $\Delta t=17^{\circ} \mathrm{C}$, and the temperature of the silicon surface facing the lamp by $\Delta t=64^{\circ} \mathrm{C}$.

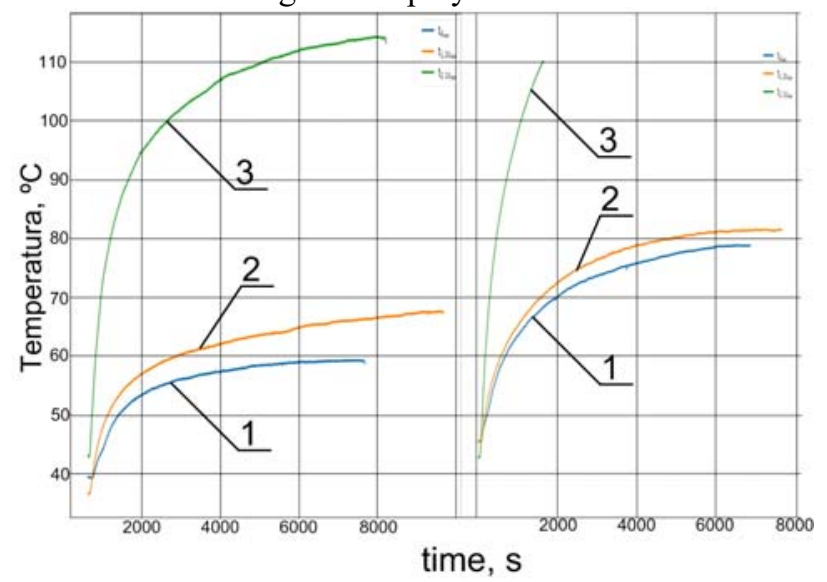

a)

b)

Fig. 6. Charts showing dependence of resistor temperature on time in the absence (a) and presence (b) of insolation with supplied voltage: $1-U_{\text {nom }} ; 2-1.2 \times U_{\text {nom }} ; 3-2.1 \times U_{\text {nom. }}$.

\section{Tests in the low voltage environmental chamber}

The environmental chamber (see Figure 7) featuring a wooden frame and insulated with PVC sheeting, comprises a structure consisting of four compartments: testing compartment, a section for generating smoke, ventilation and exit sections.
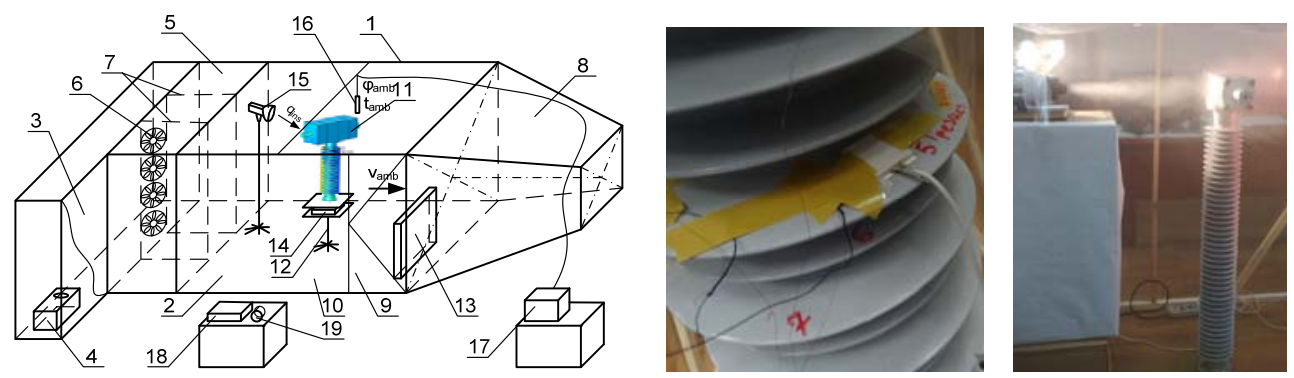

Fig. 7. Environmental chamber for low voltage tests: 1 - frame; 2 - testing compartment; 3 - smoke generation section; 4 - smoke machine ; 5 - ventilation section; 6 - fans; 7 - partitions; 8 - exit section; 9 - door; 10 - organic glass sheet; 11 - test item; 12 -support; 13 - heater; 14 -temperature measuring and control device; 15 - incandescent filament lamp for simulating insolation; 16 -primary humidity and temperature converter; 17 - thermal hygrometer; 18 - millivoltmeter; 19 - switch.

The ventilation section accommodated 4 fans for step by step control of air consumption by switching on the appropriate blowers. The test compartment was created to simulate both natural and forced convection on the surface of the combined transformer. The testing compartment accommodated a heating element to simulate air temperature, and also an incandescent filament lamp for insolation. To determine the coefficients of heat release to the ribbed surface of the resistive voltage divider we installed a heat meter (based on a thermal battery) and a differential thermocouple. We recorded heat release coefficients with natural and forced convection, surface temperature, and also the lines of airflow currents. We should note that forced convection resulted in a state of turbulent air motion (at the most frequently encountered airflow speeds). 
It was established (see Figure 8) that heat release at transformer outer surface with natural convection depends to a larger extent on the difference between the temperature at the transformer surface and ambient temperature. With forced convection, such heat release depends more on air mass speed, with greater heat release on the surface of an upward facing rib than on the surface of a downward facing rib. These data are consistent with the theory of heat transfer [4].

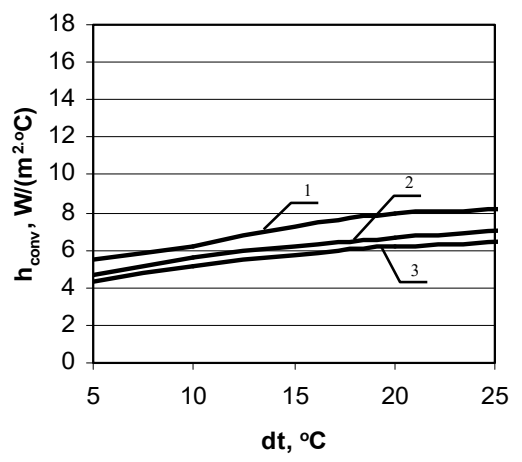

a)

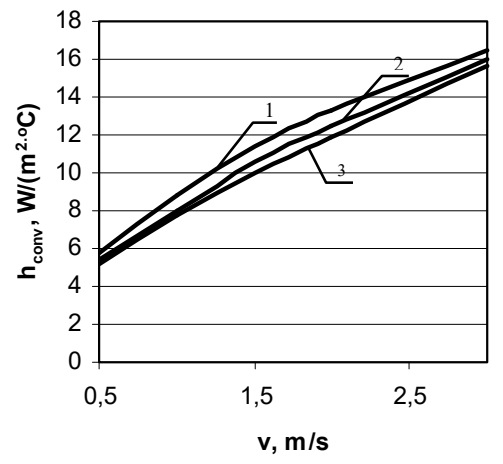

b)

Fig. 8. Dependence of convective coefficients of heat release $h_{\text {conv }}$ to surface with natural (a) and forced (b) convection: 1 - to upward facing rib surface; 2 - to cylinder; 3 - to downward facing rib.

\section{Conclusion}

The above thermal and aerodynamic research yielded experimental data necessary for the further development of a system of thermal self-diagnostics for the work of a digital combined current and voltage transformer. Insolation, voltage and air temperature significantly affect the thermal state of a transformer. The test results should be taken into account in the development of mathematical models of heat exchange between a transformer and its environment and for the development of thermal overload protection.

This research was conducted with financial support from the Ministry of Education and Science of the Russian Federation at Ivanovo State Power Engineering University within the framework of a federal target programme, "Research and Development in Priority Areas of Developing the Russian Scientific and Technological Complex in 2014-2020" concerning "A Multifunctional System based on Digital Current and Voltage Transformers for a Digital Substation" (Agreement No. 14.577.21.0276 on Granting Subsidies, dated 26 September 2017, Unique Identifier for Applied Scientific Research (Projects) RFMEFI57717X0276).

\section{References}

1. R. Hunt, M.L. Giordano, Thermal overload protection of power transformers operating theory and practical experience (Georgia Tech, Atlanta, 2005)

2. L.L. Grigsby, The Electric Power Engineering Handbook (CRC Press, Boca Raton, 2001)

3. A.A. Yablokov, N.N. Smirnov, V.V. Tyutikov, V.A. Gorbunov, MATEC Web of Conf., 141 (2017)

4. J.C. Han, L.R. Glicksman, W.M. Rohsenow, Int. J. Heat Mass Transfer, 21 (1978) 